\title{
PRÉVISIONS DE LA TEMPÉRATURE DE L'AIR EN POLOGNE JUSQU'À L'AN 2010
}

Pour désigner des trends temporels de la température de l'air au-dessus du territoire de la Pologne et exposer les prévisions devant s'étendre à des dizaines d'années les plus proches, on a eu recours à un modèle de simulation des changements naturels et anthropogéniques du climat conçus par J. Boryczka (Boryczka et al., 1992).

D'après l'auteur dudit modèle, la température de l'air $T$ est bien approximée par le trend temporel $f(t)$ de la forme

$$
f(t)=a_{0}=\sum_{r=1}^{k} b_{r} \sin \left(\frac{2 \pi}{\Theta_{r}} t+c_{r}\right)
$$

où $b_{r}-$ amplitude, $\Theta_{r}-$ période, $t-$ temps exprimé en années, $c_{r}-$ décalage de phase.

Ce trend permet une simulation de la variation cyclique de la température de l'air et on peut l'obtenir à l'aide d'une superposition des cycles contenus dans les spectres de l'oscillation d'un élément météorologique examiné.

Dans la communication présente, on a déterminé les variations cycliques de la température partant des moyennes mensuelles de la température enrégistrées de 1951 à 1990 par 58 stations météorologiques réparties également dans le territoire de la Pologne (Fig. 1).

Les périodes de la température de l'air ont été déterminées à l'aide de la méthode de Boryczka (1993) dite des "sinusoïdes de la régression". Cette méthode (conformément à la condition d'un minimum de la variance résiduelle) consiste en une approximation d'une série chronologique des résultats des mesures $y_{1}, y_{2}, \ldots, y_{\mathrm{n}}$, effectuées dans le temps $t_{1}, t_{2}, \ldots, t_{\mathrm{n}}$ à l'aide des sinusoïdes successives.

$$
y=a_{0}+b \sin \left(\frac{2 \pi}{\Theta} t+c\right)
$$

où $b$ - amplitude, $\Theta-$ période, $c-$ décalage de phase, $t-$ temps exprimé en années.

En introduisant successivement les périodes $\Theta$, on obtient le spectre de 
l'oscillation $b=b(\Theta)$ qui est une série numérique d'amplitudes $b_{1}, \ldots, b_{N}$ des sinusoïdes de la régression (par rapport au temps $t$ ) correspondant aux périodes examinées $\Theta_{1}, \ldots, \Theta_{N}$. Dans la communication présente, la période était changée chaque mois $-\Delta \Theta=1 / 12$ d'un an. Les périodes recherchées $\Theta_{r}$ sont les maxima locaux $\mathrm{R}_{r}$ d'une série numérique du coefficient de la corrélation multiple $R_{1}, \ldots, R_{n}$ auxquelles correspondent les minima de la

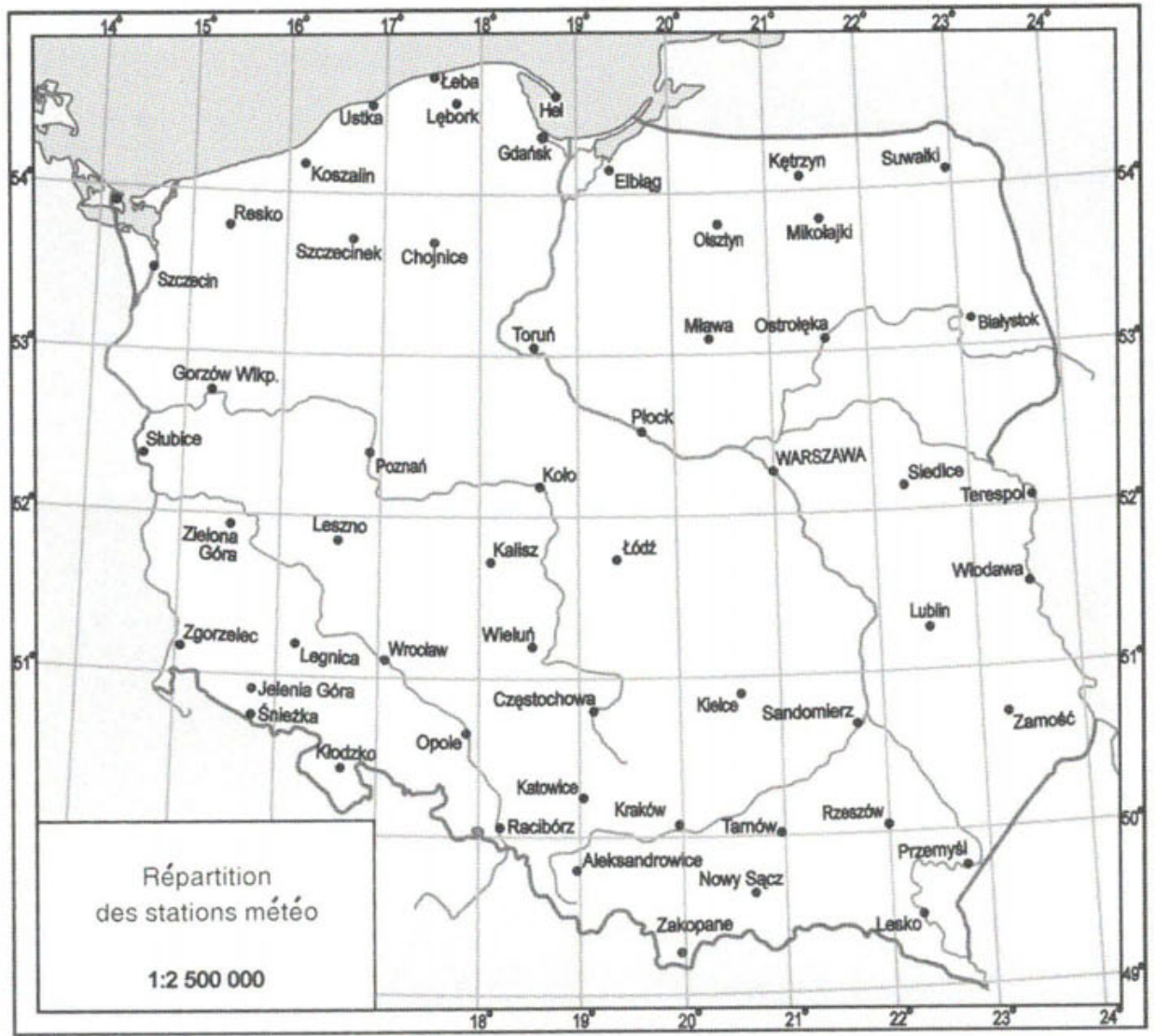

Fig. 1. Localisation des stations météorologiques.

variance résiduelle et, approximativement, les maxima des spectres denses de l'oscillation (de la série numérique des amplitudes $b_{1}, \ldots, b_{N}$ ). Les coefficients calculés de la corrélation multiple $R$ ont servi à vérifier les cycles découverts - à savoir leurs amplitudes $b_{r}$ - à l'aide du test de FisherSnedecor.

Chaque série chronologique des valeurs mesurées de la température de l'air $T_{1}, \ldots, T_{n}$ peut être considérée comme une somme de deux éléments l'élément naturel $T$ ' et l'élément anthropogénique $\Delta T$ : 


$$
T=T+\Delta T
$$

On peut distinguer l'élément naturel $T$ ' car c'est la résultante des cycles naturels qui se superposent donc ledit élément est une superposition des cycles $\Theta_{r}$ présents dans le spectre de l'oscillation:

$$
T=F(t)=a_{0}+\sum_{r=1}^{k} b_{r} \sin \left(\frac{2 \pi}{\Theta_{r}} t+c_{r}\right)
$$

Si les séries chronologiques des valeurs mesurées $T_{1}, \ldots, T_{n}$ et calculées $T_{1}^{\prime}, \ldots, T_{n}$ [comme une superposition des cycles - le trend $f(t)$ ] sont approximées à l'aide des équations des droites de la régression:

$$
\begin{aligned}
& T=A_{0}+A t \\
& T=B_{0}+B t
\end{aligned}
$$

il s'avère que la différence entre les coefficients de la régression $a=A-B$ devient une mesure des changements anthropogéniques. Le coefficient de la régression $A$ exprime une tendance résultante de la variabilité à la fois naturelle et anthropogénique (considérées comme un ensemble). Le coefficient $B$ détermine, par contre, une tendance des changements naturels, décrits à l'aide d'une superposition des cycles réels contenus dans le spectre de l'oscillation.

Autrement dit, les tendances des changements anthropogéniques de la température de l'air sont déterminées par un trend linéaire des résidus $\varepsilon_{\mathbf{i}}$ :

$$
T_{i}=T_{i}+\varepsilon_{i}, \quad i=1, \ldots, n
$$

Il ressort de l'analyse effectuée que la température de l'air au-dessus du territoire de la Pologne durant les quarante ans examinés est soumise à des variations cycliques dont les périodes varient de deux à trente ans. Les spectres denses de l'oscillation des valeurs moyennes consécutives de la température des 12 mois contiennent de 10 jusqu'à 14 rythmes $\Theta$, statistiquement importants au niveau de confiance de $95 \%$ (Fig. 2, Tableau 1 ). Le cycle le plus fort (l'amplitude $2 b$ et le coefficient de la corrélation $R$ les plus grands) observé en Pologne est le cycle de 8 ans env. Une amplitude importante (de l'ordre de $0,5-1,0^{\circ} \mathrm{C}$ ) caractérise également les cycles respectifs de 6 et 3 ans env. (apparaissant principalement dans le Sud-Est du pays). En ce qui concerne les cycles plus faibles, p.ex., les cycles d'environ 2-, 4-, 10-, 14- et même de plus de 20 ans (les variations de la température dans ces cycles sont de l'ordre de quelques dixièmes de ${ }^{\circ} \mathrm{C}$ ), on peut observer une différenciation spatiale de leurs présences respectives dans la variabilité de la température de l'air. Chacun de ces cycles est aussi différencié géographiquement (il s'agit de leurs amplitudes, périodes et phases - Żmudzka 1995). Ainsi, p.ex., dans le cycle de 8 ans environ, l'amplitude de la température de l'air varie de $0,7^{\circ} \mathrm{C}$ au sommet Kasprowy Wierch dans les Tatras à $1,6^{\circ} \mathrm{C}$ dans le Nord-Est de la Pologne, tandis que la période oscille entre 7,6 ans dans le Sud-Ouest et 7,8 ans dans le Nord-Est. 
Tableau 1

Périodes significatives $\Theta$, amplitudes $2 b$ et décalages de phase $c$ de la moyenne annuelle de la température de l'air $T$ dans les localités choisies de Pologne de 1951 à 1990.

\begin{tabular}{|c|c|c|c|c|c|c|c|c|c|c|c|}
\hline \multicolumn{4}{|c|}{ Kołobrzeg } & \multicolumn{4}{|c|}{ Warszawa } & \multicolumn{4}{|c|}{ Suwałki } \\
\hline$\Theta$ & $2 b$ & $c$ & $R$ & $\Theta$ & $2 b$ & $c$ & $R$ & $\Theta$ & $2 b$ & $c$ & $R$ \\
\hline 1,83 & 0,4 & 0,75 & 0,165 & 1,83 & 0,4 & 0,85 & 0,187 & 1,83 & 0,5 & 0,90 & 0,182 \\
\hline 2,17 & 0,4 & 2,57 & 0,173 & 2,00 & 0,4 & 1,60 & 0,174 & 2,00 & 0,5 & 1,79 & 0,185 \\
\hline 2,42 & 0,4 & 0,47 & 0,186 & 2,17 & 0,4 & 2,62 & 0,182 & 2,25 & 0,6 & $-1,47$ & 0,217 \\
\hline 2,75 & 0,4 & $-2,96$ & 0,195 & 2,42 & 0,3 & 0,36 & 0,133 & 2,42 & 0,3 & 0,74 & 0,126 \\
\hline 3,17 & 0,6 & 0,80 & 0,247 & 2,83 & 0,4 & $-0,80$ & 0,187 & 2,75 & 0,6 & $-2,54$ & 0,227 \\
\hline 3,58 & 0,6 & $-2,50$ & 0,271 & 3,17 & 0,6 & 0,90 & 0,272 & 3,17 & 0,6 & 0,84 & 0,219 \\
\hline 5,58 & 0,8 & 3,08 & 0,346 & 3,67 & 0,5 & $-1,45$ & 0,231 & 3,58 & 0,6 & $-2,44$ & 0,232 \\
\hline 7,67 & 1,4 & 1,40 & 0,599 & 5,50 & 0,8 & 2,67 & 0,334 & 4,42 & 0,3 & $-1,87$ & 0,125 \\
\hline 10,17 & 0,4 & 2,38 & 0,172 & 5,83 & 0,8 & $-1,54$ & 0,333 & 5,58 & 0,9 & 3,10 & 0,361 \\
\hline 14,00 & 0,7 & $-2,80$ & 0,306 & 7,67 & 1,5 & 1,25 & 0,633 & 7,75 & 1,5 & 1,58 & 0,587 \\
\hline \multirow[t]{3}{*}{21,83} & 0,4 & 2,83 & 0,164 & 10,25 & 0,4 & 2,24 & 0,178 & 10,17 & 0,4 & 1,64 & 0,175 \\
\hline & & & & 17,25 & 0,5 & 0,05 & 0,203 & 15,42 & 0,6 & $-1,59$ & 0,232 \\
\hline & & & & 20,67 & 0,5 & 1,92 & 0,210 & & & & \\
\hline \multicolumn{4}{|c|}{ Wroctaw } & \multicolumn{4}{|c|}{ Kasprowy Wierch } & \multicolumn{4}{|c|}{ Zamość } \\
\hline$\Theta$ & $2 b$ & $c$ & $R$ & $\Theta$ & $2 b$ & $c$ & $R$ & $\Theta$ & $2 b$ & $c$ & $R$ \\
\hline 1,83 & 0,4 & 0,67 & 0,202 & 1,83 & 0,4 & 0,43 & 0,236 & 1,50 & 0,3 & 1,08 & 0,140 \\
\hline 2,00 & 0,3 & 1,25 & 0,158 & 2,00 & 0,3 & 1,49 & 0,190 & 1,83 & 0,5 & 1,04 & 0,219 \\
\hline 2,17 & 0,4 & 2,66 & 0,171 & 2,25 & 0,3 & $-1,34$ & 0,166 & 2,00 & 0,4 & 1,69 & 0,160 \\
\hline 2,42 & 0,4 & 0,17 & 0,178 & 2,58 & 0,4 & 1,80 & 0,224 & 2,17 & 0,6 & 2,71 & 0,257 \\
\hline 2,67 & 0,4 & 2,51 & 0,182 & 2,83 & 0,3 & $-0,06$ & 0,196 & 2,83 & 0,5 & $-0,76$ & 0,208 \\
\hline 3,17 & 0,6 & 0,95 & 0,262 & 3,17 & 0,5 & 1,76 & 0,310 & 3,17 & 0,7 & 0,81 & 0,303 \\
\hline 3,58 & 0,6 & $-2,46$ & 0,259 & 3,58 & 0,7 & $-3,02$ & 0,412 & 3,58 & 0,4 & $-2,70$ & 0,196 \\
\hline 5,92 & 0,7 & $-0,92$ & 0,344 & 5,08 & 0,4 & $-0,29$ & 0,243 & 5,50 & 0,8 & 2,62 & 0,353 \\
\hline 7,58 & 1,4 & 1,10 & 0,666 & 6,00 & 0,5 & $-0,32$ & 0,290 & 7,67 & 1,3 & 1,27 & 0,582 \\
\hline 10,08 & 0,5 & 2,29 & 0,233 & 7,67 & 0,7 & 1,16 & 0,429 & 10,33 & 0,5 & 2,42 & 0,223 \\
\hline 14,08 & 0,4 & $-2,99$ & 0,194 & 10,17 & 0,4 & 2,41 & 0,224 & 16,00 & 0,6 & $-1,07$ & 0,263 \\
\hline \multirow[t]{2}{*}{20,92} & 0,4 & 2,01 & 0,159 & 13,67 & 0,4 & $-2,75$ & 0,222 & & & & \\
\hline & & & & 28,08 & 0,4 & $-1,34$ & 0,257 & & & & \\
\hline
\end{tabular}

Les trends temporels $f(t)$ ont été obtenus à l'aide d'une superposition de dix cycles naturels; pour chaque station météorologique, les cycles les plus forts (avec le coefficient de corrélation multiple $R$ le plus grand) ont été choisis parmi les cycles statistiquement importants. Ces trends se caractérisent par des coefficients de corrélation $R$ assez grands (l'approximation d'une grande exactitude) et par des erreurs standards $\delta$ peu importantes. Tous ces trends sont significatifs au niveau de confiance de $95 \%$ (Tableau 2). 

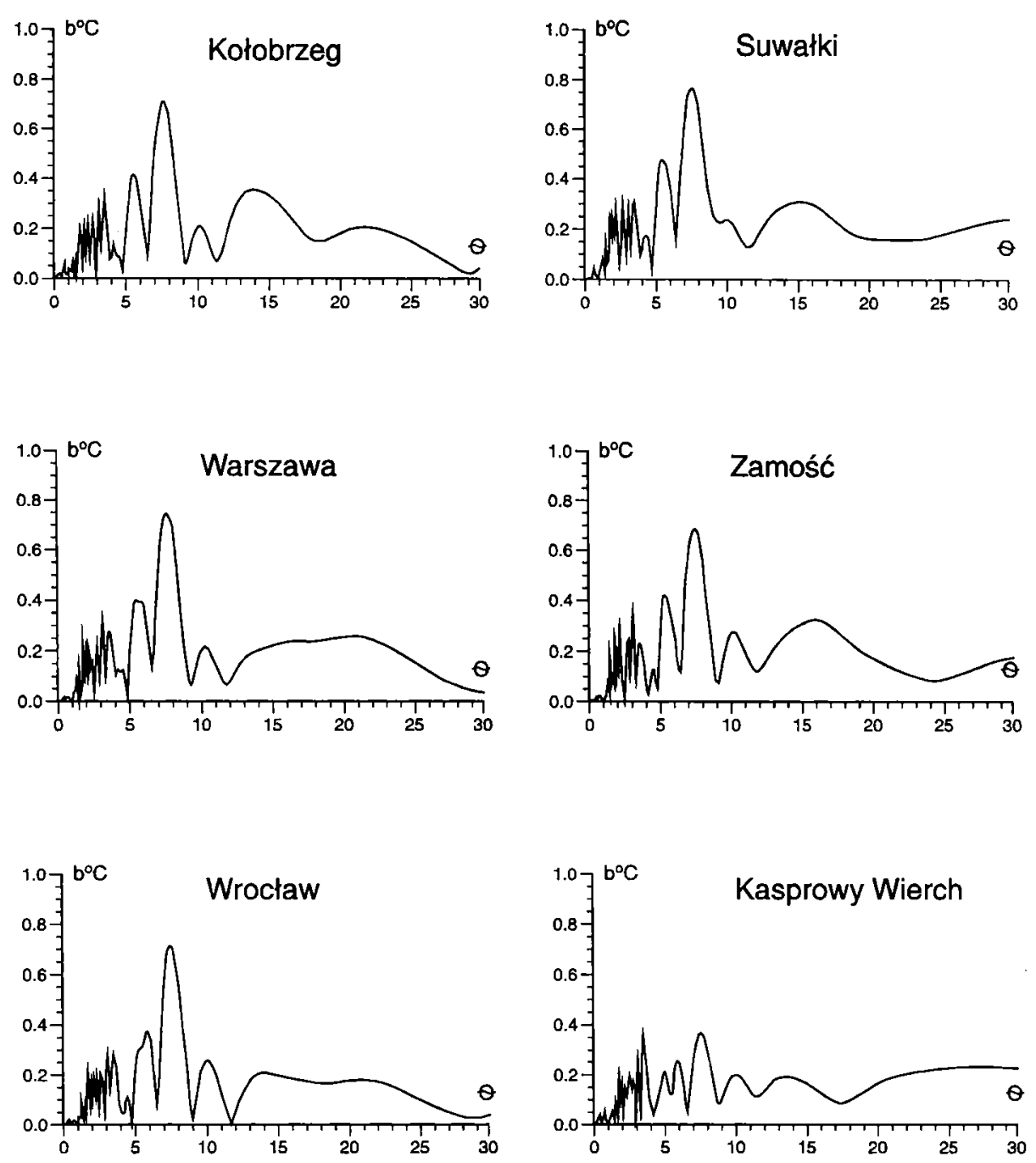

Fig. 2. Spectres de l'oscillation de la moyenne annuelle de la température de l'air, dans les localités choisies de Pologne (1951-1990).

Comme il a été mentionné plus haut, cette approximation est d'une grande exactitude - la preuve en sont les différences peu importantes $(0,0$ - 0,3 deg) entre les valeurs mesurées de la température et celles calculées à partir des fonctions des trends. Cette comparaison a été effectuée pour une décennie 1981-1990.

Une superposition des dix cycles naturels $\Theta$ (contenus dans les spectres de l'oscillation dans les points de mesure particuliers) nous conduit aux équations suivantes des droites de la régression $T^{\prime}=B_{0}+B t$ (Tableau 3). 
Estimation statistique des trends temporels $f(t)$

\begin{tabular}{|l|c|c|c|c|}
\hline$f(t)$ & $\varepsilon$ & $\delta$ & $R$ & $F$ \\
\hline Kołobrzeg & 0,38 & 0,39 & 0,88 & 80,27 \\
Suwałki & 0,41 & 0,42 & 0,89 & 83,19 \\
Wrocław & 0,35 & 0,36 & 0,88 & 80,62 \\
Zamość & 0,37 & 0,38 & 0,89 & 88,58 \\
\hline
\end{tabular}

Equations des droites de la régression de la température de l'air $T^{\prime}$ (calculée de la fonction des trends $f(t)$ par rapport au temps $t$

\begin{tabular}{|l|c|c|c|}
\hline Localité & $T^{\prime}=B_{0}+B t$ & $R$ & $F$ \\
\hline Kołobrzeg & $T^{\prime}=7,56+0,011 \cdot t$ & 0,17 & 13,54 \\
Suwałki & $T^{\prime}=5,99+0,003 \cdot t$ & 0,04 & 0,88 \\
Wrocław & $T^{\prime}=8,21+0,005 \cdot t$ & 0,09 & 3,54 \\
Zamość & $T^{\prime}=7,19+0,003 \cdot t$ & 0,05 & 1,33 \\
\hline
\end{tabular}

Les tendances des changements naturels de la moyenne de la température de l'air dans les localités choisies, de 1951 à 1990, sont les suivantes:

Kołobrzeg $B=0,11 \mathrm{deg} / 10$ ans

Suwałki $\quad B=0,03 \mathrm{deg} / 10$ ans

Wroclaw $B=0,05 \mathrm{deg} / 10$ ans

Zamość $\quad B=0,03 \mathrm{deg} / 10 \mathrm{ans}$

Par contre, les équations des droites de la régression qui approximent les séries chronologiques des valeurs mesurées de la température de l'air ont donné des valeurs suivantes du coefficient de la régression $A$ :

Kołobrzeg $A=0,28 \mathrm{deg} / 10$ ans

Suwałki $A=0,02 \mathrm{deg} / 10$ ans

Wrocław $A=0,11 \mathrm{deg} / 10$ ans

Zamość $\quad A=0,02 \mathrm{deg} / 10$ ans

Ainsi, l'élément anthropogénique $(a=A-B)$ peut contenir les valeurs allant de $-0,01 \mathrm{deg} / 10$ ans à Zamość et Suwałki jusqu'à $0,06 \mathrm{deg} / 10$ ans à Wrocław, et $0,17 \mathrm{deg} / 10$ ans à Kołobrzeg.

Les valeurs positives du coefficient de la régression $A$ montrent que, dans les années 1951-1990, la Pologne a connu un réchauffement progressif. Comme nous l'avons expliqué plus haut, ce phénomène dépend aussi bien des changements naturels (coefficient de la régression $B$ ) que des changements anthropogéniques $(a=A-B)$. Dans l'Est du pays, une augmentation de la température de l'air résulte principalement des changements naturels, décrits à l'aide d'une superposition de cycles de cette température. Dans l'Ouest, par contre, l'apport de l'élément anthropogénique est plus significatif. 


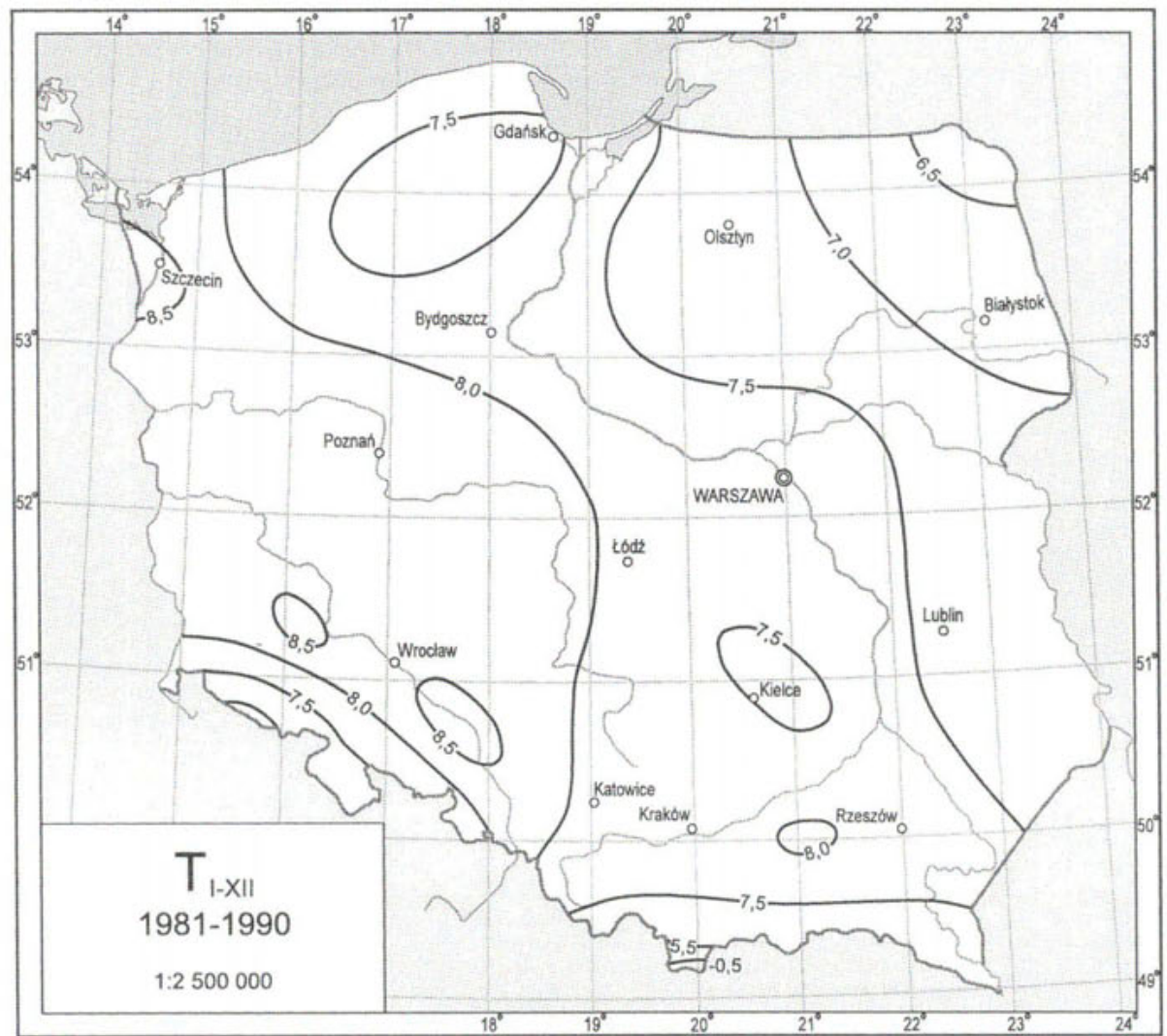

Fig. 3a. Moyenne annuelle de la température de l'air, 1981-1990.

Nous nous sommes servis des trends temporels $f(t)$ pour établir les prévisions des variations de la température de l'air $(t>n$ où: $t-$ temps en années $/ t=0$ en 1950/, $n$ - longueur de la série chronologique). Les segments des courbes des changements de la température faisant l'objet des prévisions s'élèvent à $t=41-60$ (1991-2010). Les prévisions ne s'étendent qu'à la vingtaine d'années la plus proche étant donné que dans les fonctions des trends temporels n'ont été utilisés que les cycles courts (périodes de 1 à 35 ans). Dans les sondages se rapportant à l'avenir et comportant des fractions de temps plus longues, il faudrait prendre également en considération une variabilité à long terme dont l'influence sur la formation de la température en Pologne est importante (Boryczka, 1993).

Ayant admis que les extrêmes des cycles découverts de la température de l'air se répéteraient dans l'avenir, on a extrapolé les valeurs des fonctions obtenues $f(t)$ en calculant les moyennes annuelles de la température en question pour les décades successives, et notamment 1991-2000 et 2001-2010. Les prévisions des valeurs moyennes de la température pour 


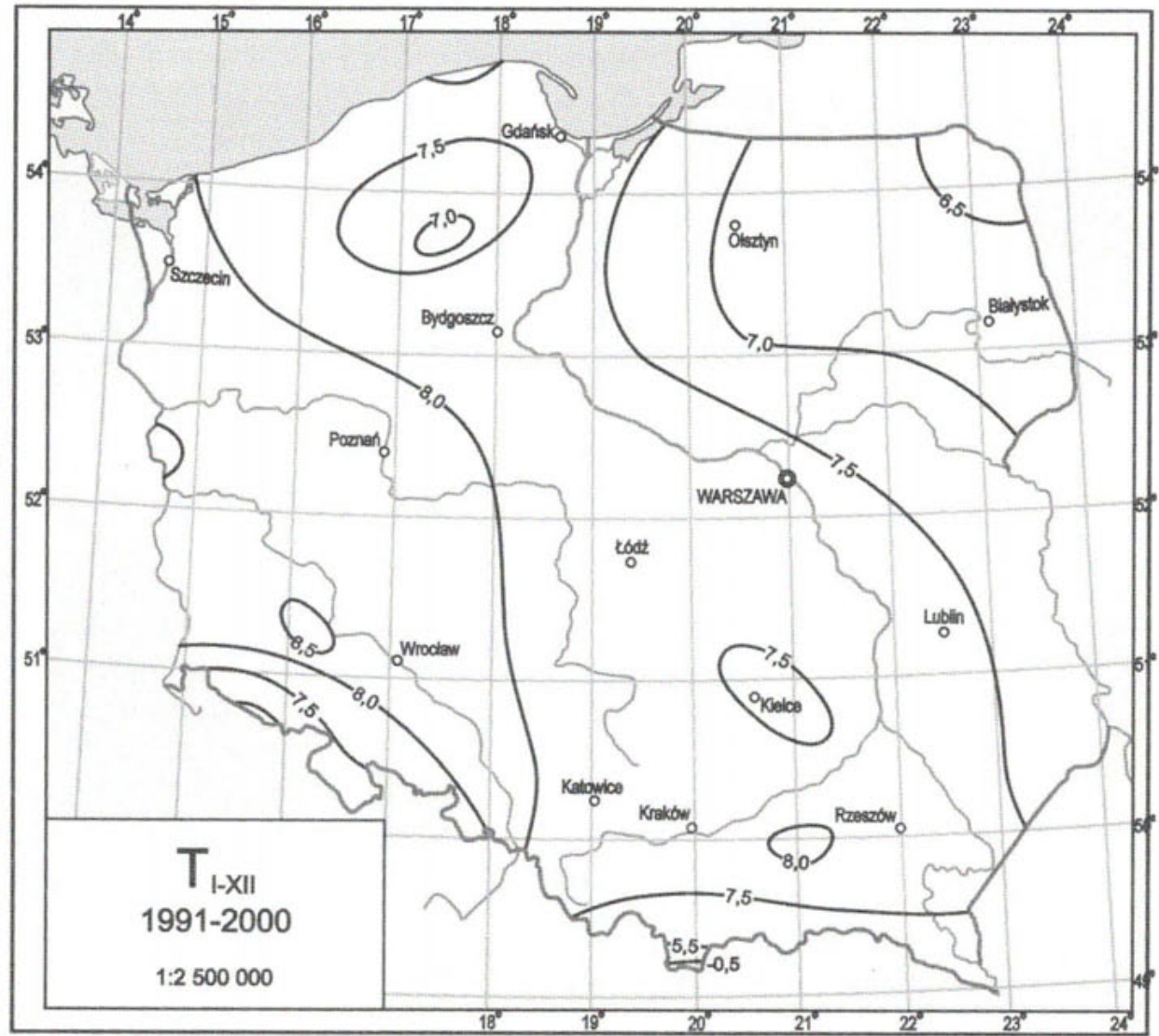

Fig. 3b. Moyenne annuelle de la température de l'air, 1991-2000.

une période de 10 ans sont plus dignes de foi que celles relatives aux moyennes annuelles.

La distribution des résidus $\varepsilon_{\mathrm{i}}\left(\varepsilon_{\mathrm{i}}=T_{\mathrm{i}}-f\left(t_{i}\right)\right.$ dans la population est proche de la distribution normale aux paramètres $0, \delta$, où $\delta$ est une erreur standard.

Les seuils de confiance des trends hypothétiques $f(t)$ (dans la population) au niveau de confiance de $95 \%$ sont

$$
f(t)-1,96 \delta<f(t)<f(t)+1,96 \delta,
$$

où $\delta$ sont les erreurs standards des fonctions approximantes $f(t)$ (Tableau 2).

Citons, à titre d'exemple, les seuils de confiance des trends hypothétiques $T=f(t)$ au niveau de $95 \%$ qui, dans les localités ci-dessous, s'élèvent à:

Kołobrzeg $f(t) \pm 0,76^{\circ} \mathrm{C}$,

Suwałki $f(t) \pm 0,82^{\circ} \mathrm{C}$,

Wrocław $f(t) \pm 0,69^{\circ} \mathrm{C}$,

Zamość $\quad f(t) \pm 0,74^{\circ} \mathrm{C}$. 


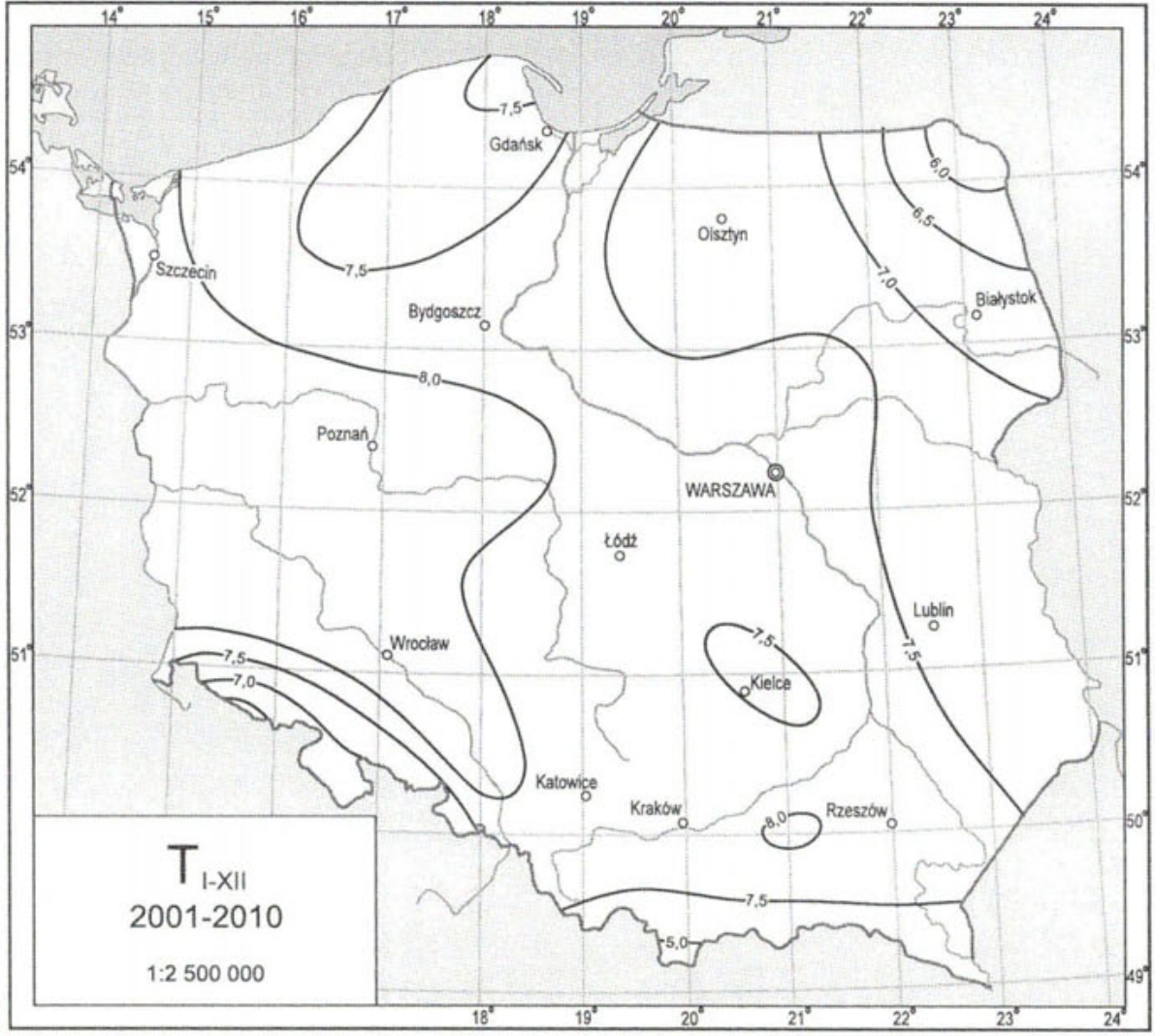

Fig. 3c. Moyenne annuelle de la température de l'air, 2001-2010.

Les données ci-dessus signifient que les valeurs de la température de l'air (moyennes consécutives des 12 mois) mesurées au début du XXI ${ }^{\mathrm{e}} \mathrm{s}$. devraient se situer entre les seuils présentés plus haut avec une probabilité de $95 \%$.

Il ressort des prévisions obtenues que les régularités générales de la distribution de la température dans les décades successives n'auront pas subi des changements. Les isothermes conserveraient leur forme actuelle bien qu'on puisse y apercevoir des différences peu importantes. Comparé à la période 1981-1990, l'espace compris dans l'isotherme $8,0^{\circ} \mathrm{C}$ se serait diminué au cours des deux décades successives. Dans les années 20012010, la moyenne annuelle de la température de l'air ne devrait pas dépasser $8,5^{\circ} \mathrm{C}$ dans le territoire de la Pologne, tandis que dans les confins Nord-Est du pays se manifesteraient probablement les valeurs moindres que $6,0^{\circ} \mathrm{C}$ (Fig. $3 \mathrm{a}, \mathrm{b}, \mathrm{c}$ ).

Les résultats de recherches indiquent la possibilité d'un refroidessement naturel dans tout le territoire du pays, dans les vingt ans à venir. Ce refroidissement résulterait d'une superposition des cycles naturels de la 
température, lesdits cycles, comme on a démontré (Boryczka, 1997), étant corrélés avec les changements respectifs de la circulation atmosphérique, de l'activité solaire, de l'activité volcanique et des paramètres du Système solaire. D'un autre côté, une augmentation anthropogénique de la température qui ne cesse de progresser (relative à une tendance croissante de la concentration du $\mathrm{CO}_{2}$ dans l'atmosphère) et qui est considérée comme une caractéristique réelle du climat actuel aussi bien de la Pologne que de l'Europe (Kożuchowski, 1996) freinerait le refroidissement en question.

Dans la communication présente, on s'est servi de la moyenne annuelle de la température de l'air exprimant assez bien la tendance générale des changements climatiques. Tout de même, les conclusions plus précises, surtout se rapportant aux causes des changements en question, devraient être formulées à partir des données qui tiennent compte de la variabilité saisonnière de la température de l'air.

\section{BIBLIOGRAPHIE}

Boryczka J., Stopa-Boryczka M., Kicińska B., Żmudzka E., 1992, Atlas wspótzależności parametrów meteorologicznych i geograficznych w Polsce, t. 7 , Zmiany wiekowe klimatu Polski (Atlas des corrélations des paramètres météorologiques et géographiques en Pologne, vol. 7, Changements séculaires du climat de la Terre), Warszawa.

B or y c z k a J., 1993, Naturalne $i$ antropogeniczne zmiany klimatu Ziemi $w$ XVII-XXI $w$. (Changements naturels et anthropogéniques du climat de la Terre, entre les $\mathrm{XVII}^{\mathrm{e}}$ et XXI $\mathrm{XI}^{\mathrm{e}}$ ss.), Warszawa.

Ko ż u chowski K., 1996, Współczesne zmiany klimatyczne w Polsce na tle zmian globalnych (Changements climatiques contemporains en Pologne comparés aux changements globaux), Przeglad Geograficzny, 68(1-2), Warszawa.

$\dot{\mathrm{Z}} \mathrm{mudzka}$ E., 1995, Tendencje i cykle zmian temperatury powietrza w Polsce w latach 1951-1990 (Tendences et cycles des changements de la température de l'air en Pologne en 1951-1990), Przeglad Geofizyczny, 40(2), Warszawa. 